\title{
A Survey on Organization and Positioning of Nodes in Wireless Sensor Network
}

\author{
Xinyi Tan , Fuquan Zhang*, MengMeng Shi, Demin Gao and Yunfei Liu \\ College of Information and Sciences \\ NanJing Forestry University \\ NanJing, China \\ E-mail: zfq@njfu.edu.cn; dmgao@njfu.edu.cn; lyf@njfu.edu.cn
}

\begin{abstract}
Wireless sensor network has a rapid growth in both science research and commercial development as well as been widely used in a variety of realms. Facing different kinds of challenging issues of WSNs, the deployment of nodes stands out for it has a critical influence on area or target coverage, network connectivity, network lifetime, etc. This paper explores classifications of node and deployment strategies from different angles, weighing their pros and cons and analyzing its impacts on the performance of a WSN.
\end{abstract}

Keywords: wireless sensor network, WSN, deployment

\section{Introduction}

A wireless sensor network (WSN) is generally described as a network of nodes that corporately monitor physical of environmental conditions such as temperature, vibration, pressure, pollutants, humidity or light and collect information about the observed phenomena or events. It has limited energy and processing capabilities and its topology changes over time due to nodes running out of energy. Basically most of the WSN has a OSI model with five layers: application layer, transport layer, network layer, data link layer and physical layer [1].

According [2], the WSN market will grow to $\$ 1.8$ billion by 2024 . It has been employed in a large number of diverse application domains and new application areas keep emerging. Typical applications of WSNs include, but not limited to [3][4]:

- Military Applications: Detection, classification, and tracking of intruders/targets/objects, for example, submarine movements, battlefield surveillance, enemy intrusion, are a basic surveillance or military application of WSNs and have been studied in the literature from many aspects.

- Environment Applications: Forecast and detection of weather or disasters, observation or tracking of animals are the purposes of this kind of applications are designed for.

- Industry and Agriculture Applications: For precision agriculture and the safety and efficiency of factories, sensor nodes are being deployed to monitor conditions of plants or stock, check security elements or inventories.

- Urbanization and Infrastructure: A kind of applications used for bridge/tunnel/highway monitoring, water or air quality monitoring, traffic management, crime prevention and smart home/buildings.

- Health Applications: It is a promising technologies, including patient monitoring, sports evaluation, artery exploration, etc.

In a WSN, deployment is an approach by which sensor nodes are organized and placed in the given area to achieve certain goals. It determines parameters and affects many aspects of the network in a large extent. To get a fully understanding of its importance and 
basic strategies, the rest of the paper is organized as follows: the next section is dedicated to introduce the things that are deployed-nodes. Section 3 discusses objectives of an optimal deployment and in section 4, we present different strategies of deployment classified according to a range of criteria and evaluate their performance. Finally, Section 5 concludes the paper.

\section{Wireless Sensor Nodes}

A wireless sensor node is the unit of a WSN with five major components: a microcontroller, transceiver, external memory, power source and one or more sensors. that enable it to sense, process and communicate. However, its processing, storage and communication capabilities are limited. We classified nodes into different categories of various types.

\subsection{Basic Categories}

For any beginning learner of a WSN, nodes are divided into four types: sensing nodes, relay nodes, cluster heads and base stations.

- Sensing node: A sensing node can monitor a region or target of interest and transmit the collected information. It is the most crucial part of a WSN and the main component.

- Relay node: To eliminate the routing load unbalance among sensing nodes, one approach is to deploy a small number of relay nodes acting as routing nodes in WSNs. These relay nodes, participating in forwarding of the information towards the final destination, often have stronger energy supply, communication capability and computation capability, but also costlier. Positioning of relay nodes has been considered as a means for establishing an efficient network topology [5].

- Cluster head: A scalable WSN will be partition into several disjoint parts called clusters, each of which has one designated cluster head and several cluster members. Cluster heads usually collect and aggregate the data in their individual clusters and often conduct cluster management duties consistent with their capabilities. When empowered with sufficient computational resources, cluster heads can create and maintain a multi-hop intra-cluster routing tree, arbitrate medium access among the sensors in the individual clusters, assign sensors to tasks, etc. To provide efficient communication, cluster heads need multiple radios so they can communicate with multiple nodes simultaneously. Also, to ensure sufficient power supply, nodes in a cluster could take turns in assuming the role of cluster head. Careful positioning of cluster heads in a WSN has been deemed an effective strategy for establishing an efficient network topology.

- Base station: A base station is a transceiver where sensing data are collected and aggregated at. It can be connected to a designated Web server that makes it possible for authorized users to remotely access and configure the WSN anytime, anywhere via traditional computers or mobile devices. All nodes are supposed to connected to a base station either directly or over a multi-hop path for sending the sensed data. Most published approaches for cluster heads placement apply to base stations for the same network model, and vice versa. The similarity is mostly due to the fact that both cluster heads and base stations collect the data from sensor nodes and take some leadership role in setting up and managing the network.

Since cluster heads and base stations often act as data collection agents for sensors, we collectively refer to them as data collectors. 


\subsection{Static Nodes and Mobile Nodes}

According to whether a node has the movement capability, wireless sensors can be divided into fixed nodes and mobile nodes.

- Static node: A static node, also be known as a fixed or stationary node, has the ability to collect sensed data, send or receive messages, process data and messages and do other types of computation in WASNs. Typically, these sensor nodes do not move once they are deployed.

- Mobile node: A mobile node not only has all the features of the fixed nodes, but also has some mobility. A mobile node can act as a router when it is in a low or even no coverage area, and accomplish the recovery task. It only moves logically and sometimes designates the fixed sensor nodes as their proxies.

\subsection{Coverage Nodes and Connectivity Nodes}

Sensing coverage and network connectivity are two of the most fundamental issues to ensure effective environmental sensing and robust data communication in a WSN application, which have a great impact on QoS of WSNs [6]. Thus, nodes can be logically divided into two types: coverage nodes and connectivity nodes.

- Coverage node: Deploy coverage node at the first stage is to achieve a relatively high network coverage rate.

- Connectivity node: Then, place connectivity node to ensure full network connectivity.

Coverage and connectivity will be introduced in the next section as objectives for deployment.

\subsection{Active Node and Sleep Nodes}

Energy is paramount concern in WSN applications that need to operate a long time with the limited battery power that usually cannot recharge. An effective approach for energy conservation is scheduling sleep intervals for some nodes, while the remaining nodes stay active to provide continuous service in different rounds.

- Active node: When at least one component or all the components of a node are turned on, operations such as sensing or collecting information, data processing, message sending or receiving must consume energy and that node is an active node.

- Sleep node: If the whole components of a node are turned off but a timer or some other triggering mechanism may be running, it is called a sleep node.

\subsection{Sensors and Actuators}

Resent years have arisen the wireless sensor and actuator network (WSAN), which combines normal sensors with actuators, that can take actions adds a new dimension to the WSN paradigm. They communicate and collaborate to perform distributed sensing and acting tasks [7].

- Sensor: A sensor can gather information while monitoring a given environment or state of a system.

- Actuator: Actuators are able to change parameters in their environment by performing actions that affect the environment. They enable a WSN to participate in controlling their surroundings.

\subsection{Regular Nodes and Beacons}

From the view point of localization systems, we have two types of nodes: regular nodes and beacons. Regular nodes refer to nodes in the network that have no knowledge of their position and no special hardware to acquire this information. Beacon nodes, also known 
as land-marks or locators, are nodes that do not require a localization system to estimate their physical positions. In fact, they form the base of these systems. Their position is obtained by manual placement or external means such as a global positioning system (GPS).

\section{Objectives of an Optimal Deployment}

Application developers surely like the sensors to be deployed in a way that aligns with the overall design goals. Therefore, most of the proposed node placement schemes in the literature have focused on maximization of coverage, achieving strong network connectivity, minimization of energy consumption and boosting data fidelity. A number of secondary objectives such as tolerance of node failure, load balance, minimization cost of deployment and communication overheads have also been considered.

\subsection{Maximization of Coverage}

Coverage can be defined as how well or to how much extent each point of a deployed network is under the vigilance of a sensor node [8]. Maximal coverage of the monitored area is the objective that has received the most attention in the literature, which reflects how well and how long the area of sensor field is monitored [9]. Two aspects should be considered about coverage: whether the initial layout of sensor nodes covers the whole target area; whether these nodes collect complete and accurate information in the target area.

Simply given the case of two dimensions, according to the different monitoring areas, four types of coverage have been defined [10]:

\subsubsection{Regional Coverage}

In regional coverage, each point is under the surveillance of at least one sensor. Since each point requires monitoring, in this type of coverage the sensing nodes are densely deployed and which results into overlapped coverage.

\subsubsection{Point Coverage}

Under the point coverage only a limited number of discrete objects or target points are to be monitored. In this type of coverage, the sensor nodes are divided into node subsets under the stochastic distribution. Due to such type of division each subset works turn by turn which results into maximization of the network lifetime.

Any point is said to be $\mathrm{k}$-covered if it is within sensing ranges of at least $\mathrm{k}$ sensors. A network is said to have $\mathrm{k}$-coverage if each point in it is covered by at least $\mathrm{k}$ sensors [11].

\subsubsection{Barrier Coverage}

A barrier is a line of sensors across the entire field of interest. The sensing ranges of two neighbor sensors in the barrier are overlapped and thus the intruder are guaranteed to be detected. In the applications, the sensors are always deployed outdoors to achieve barrier coverage[12]. Barrier coverage deals with the chance calculation of movement of objects in the targeted area. The chances or probability can be determined by movement rate of object and sensing intensities of the sensors for each and every point on the path followed by the object. On the basis of this calculation, the density of sensor nodes can be computed. It can be find out that how densely the sensor nodes should be deployed in the targeted region. Barrier coverage further can be classified as Weak k-barrier and Strong kbarrier coverage. 
- Weak k-barrier coverage: This type of coverage guaranteed that any target crossing a region along with orthogonal path is detected by at least k-sensors.

- Strong k-barrier coverage: This coverage guaranteed that target is detected by ksensors, however, the path followed by the target does not matter.

\subsubsection{Path Coverage}

In this coverage, the goal is to minimize or maximize the probability of undetected penetration through the region.

\subsection{Network Connectivity}

Along with coverage, the notion of connectivity is equally important in wireless sensor networks. A WSN must provide satisfactory network connectivity, so as to eliminate the isolation of sensors and enable each sensor to communicate successfully. By a connected network we mean that between any two nodes there exists a single-hop or multi-hop communication path. The WSN connectivity is primarily determined by the deployment locations, the number and the communication ranges of nodes.

If connected paths between any two nodes is no less than $\mathrm{k}$, it can be defined as $\mathrm{k}$ connectivity [13]. K-connectivity can enhance robustness of WSNs by making sure they may still continue to work even of damage or failure of some nodes.

In WSNs, connectivity acting as a fundamental property helps in the following ways:

- Connectivity represents the network status on the basis of network density, node deployment plans, sensing and communication ranges.

- Connectivity provides the roadmap for development and working plan for a network such as deployment patterns, processing algorithms etc.

K-connectivity implies that there are $\mathrm{K}$ independent paths among every pair of nodes. For $\mathrm{K}>1$, the network can tolerate some node and link failures and guarantee certain communication capacity among nodes.

\subsection{Minimization of Energy Consumption}

Since sensors typically operate on batteries and are thus limited in their active lifetime, the minimization of energy consumption has been a critical concern in WSN applications. It can be obtained by achieving energy efficient and energy balance [14], that is, maintaining both network coverage and connectivity with minimum active node.

The positions of nodes significantly impact the network lifetime. For example, variations in node density throughout the area can eventually lead to unbalanced traffic load and cause bottlenecks. In addition, a uniform node distribution may lead to depleting the energy of nodes that are close to the base station at a higher rate than other nodes and thus shorten the network lifetime. Routing protocols and node scheduling are two other important aspects of the network lifetime because they significantly impact the overall energy dissipation.

Basically, optimization deployment, select the routes and the corresponding power levels [15], effective transmission of data, scheduling sleep intervals and relocating of DCs are key issues to reduce energy consumption and prolong network lifetime.

\subsection{Boosting Data Fidelity}

Ensuring the credibility of the gathered data is obviously an important design goal of WSNs. A sensor network basically provides a collective assessment of the detected phenomena by fusing the readings of multiple independent (and sometimes heterogeneous) sensors. Data fusion boosts the fidelity of the reported incidents by 
lowering the probability of false alarms and of missing a detectable object. From a signal processing point of view, data fusion tries to minimize the effect of the distortion by considering reports from multiple sensors so that an accurate assessment can be made regarding the detected phenomena. Increasing the number of sensors reporting in a particular region will surely boost the accuracy of the fused data [16].

\section{Strategies of Deployment}

Deployment of nodes in WSNs refers to the placement of nodes to forming the desired network. The goal of node deployment is to determine the suitable position of nodes to increase sensor node coverage, enhance global connectivity, improve data accuracy and extend network lifetime at a relatively low cost. Sensor deployment strategies play a very important role in providing better quality of service (QoS) for applications, thus it must be planned considerably. Sensor deployment must take into account the nature of the terrain, for example obstacles such as buildings and trees in the line of vision for sensors, uneven surfaces and elevations for hilly terrains, redundancy due to the likelihood of sensor failures, and the power needed to transmit information between deployed sensors and between a deployed sensor and the cluster head [17]. The sensor node deployment can be divided into static, mobile, and mixed-sensor deployment according to whether there are mobile nodes in WSNs or all of them are static nodes or mobile nodes.

\subsection{Static Deployment}

Static deployment is an approach by which the locations of nodes do not change after they were placed in the area to be monitored. So we can get exact layout of the nodes until some nodes run out of energy. This scenario includes industry and agriculture applications, urbanization and infrastructure application, health application, etc. Static deployment is divided into two categories according to the accessibility of the monitored area, costs of deployment and objectives of applications, (1) deterministic deployment in a controlled and human-friendly environment with relatively expensive sensors and (2) random deployment in a dangerous and inaccessible region with cheaper sensors [18].

\subsubsection{Deterministic Deployment}

The deterministic deployment, also known as controlled deployment, is to deterministically place the sensor nodes in order to meet the desired performance goals. It is widely used in scenarios where nodes need to be installed at the exact location, such as populating an area with highly precise seismic nodes, underwater applications, bridges, roads and indoor applications, etc. Deployment enables nodes to meet coverage and connectivity requirements, therefore, careful analysis and design for these applications must be done. Deterministic node deployment is viable and often necessary when sensors are expensive or when their operation is significantly affected by their position.

The locations of the nodes can be predetermined and hence can be hand-placed or deployed using mobile robots. Sensors are deliberately and precisely placed at desirable locations in a controlled fashion following regular patterns. Popular patterns including square, triangle, and hexagon that can be repeated to cover a continuous region without having any overlapping areas are widely adopted in practice due to the simplicity and the convenience of deployment. Other patterns such as rhombus and mutated patterns are also investigated in the literature in terms of minimizing the required number of sensors while providing desirable quality of service QoS for various application contexts.

\subsubsection{Random Deployment}

In other cases, if the location of most nodes cannot accurately determined, the only option is random deployment. These scenarios are particularly true for military 
applications like reconnaissance missions during combat, environment application such as forest fire monitoring, disaster recovery and so on. When the price of sensor nodes is cheaper and the area is dangerous, this approach is rational and feasible. In these applications, density of the sensor is an important parameter.

Random deployment is appealing for large-scale WSN applications, so it is widely expected that sensors will be dropped by helicopters or ships, grenade launchers or clustered bombs. Such means of deployment lead to random spreading of sensors; although the node density can be controlled to some extent. However, random placement does not guarantee full coverage because it is stochastic in nature, hence often resulting in accumulation of nodes at certain areas in the sensing filed but leaving other areas deprived of nodes.

Generally speaking, the WSNs resulting from deterministic sensor deployment provide better sensing coverage and higher degree of connectivity, comparing to the random counterparts. On the other hand, it was found in that deterministic sensor deployment does not always outperform the random sensor deployment for applications that do not require full coverage such as intrusion detection.

\subsection{Mobile Deployment}

In contrast to static deployment, mobile deployment using nodes with the capability of moving or robots to carry nodes. Such networks usually are capable of self-deployment starting from an initial configuration, namely randomly throw nodes firstly, and then using a selection of optimization algorithm for deployment optimization. Such as Virtual force algorithm, virtual force oriented particles algorithm, simulated annealing algorithm, particle swarm optimization algorithm and simulated annealing genetic algorithm. The nodes would spread out such that coverage in the sensing field is maximized while maintaining network connectivity.

Obviously, dynamic changes happen during the network operation. For example, traffic patterns can change based on the monitored events, or the load may not be balanced among the nodes, causing bottlenecks. Also, application- level interest can vary over time, and the available network resources may change as new nodes join the network, or as older nodes run out of energy. Therefore, moving nodes while the WSN is operational is necessary to further improve the performance of the network. For instance, when many of the sensors in the vicinity of a DC stop functional due to the exhaustion of their batteries, some redundant sensors from other parts of the monitored region can be identified and relocated to replace the dead sensors or the DC can move to a new place in order to improve the network lifetime. Such dynamic relocation can also be very beneficial in a target tracking application where the target is mobile. For instance, some of the sensors can be relocated close to the target to increase the fidelity of the collected data. Moreover, in some applications it may be wise to keep the DC a safe distance from harmful targets, e.g., an enemy tank, by relocating it to safer areas, its availability is ensured.

\subsection{Mixed-sensor Deployment}

Though mobile deployment does create a more efficient WSN, the sole use of mobile nodes would not be practical due to the increased cost and management overhead.

Research has also been conducted in mixed-sensor networks, where some of the nodes are mobile and some are static. The deployment of nodes in mixed-sensor networks, which require one to strike a balance between the number of static and mobile sensors, involves the optimization of a cost/performance-based objective function and is therefore challenging.

Initially a fixed number of static nodes are deployed, which deterministically find the exact amount of coverage holes existing in the entire network using the structure of Voronoi diagrams and then dynamically estimate the additional number of mobile nodes 
needed to be deployed and relocated to the optimal locations of the holes to maximize overall coverage. This approach of deploying a fixed number of static nodes and a varying estimated number of mobile nodes can provide optimal coverage under controlled cost.

The mixed-sensor deployment is the most effective and efficient placement strategy for large-scale and mission-critical WSN, because it allows one to choose the degree of coverage required by the underlying application as well as gives an opportunity to optimize on the number of additional mobile nodes needed to be deployed.

\subsection{Static and Dynamic Deployment}

The deployment strategies can also be categorized into static and dynamic depending on whether the optimization is performed at the time of deployment or while the network is operational, respectively and they are applicable to only mobile sensor networks.

Since random placement does not guarantee full coverage because it is stochastic in nature, hence often resulting in accumulation of nodes at certain areas in the sensing field but leaving other areas deprived of nodes. Approaches are also proposed to detect coverage holes after an initial deployment and to try to heal or eliminate those holes by moving sensors to those locations, achieving maximum coverage. The whole process is called optimization.

Optimal node placement is a very challenging problem that has been proven to be NPHard for most of the formulations of sensor deployment. Relocating the nodes during regular network operation is even more challenging compared with the static deployment for it requires continual monitoring of the network state and performance as well as analysis of events happening in the vicinity of the node otherwise it can potentially cause disruption in data delivery.

\subsection{Indoor and Outdoor Deployment}

Depending on where nodes are placed, deployments are classified in three categories; (1) outdoor, (2) indoor, and (3) indoor and outdoor. Indoor and outdoor deployment is wildly used in environment monitoring applications for humidity or air quality and smart building/home. Usually, outdoor environments require a large number of nodes to cover a large area whereas fewer nodes are sufficient for indoor environments to form a network in a limited space[19]. Existing WSN based environment monitoring systems can also be categorized into indoor, outdoor, and greenhouse based on their deployment areas [20].

\subsection{Two-dimensional and Three-dimensional Deployment}

According to whether nodes are placed in a two-dimensional plane or a threedimensional space, deployment can be classified into two-dimensional deployment and three-dimensional deployment. In most of the applications, two-dimensional deployment of wireless sensor nodes is assumed, where height is considered negligible, like in terrestrial networks. A point is covered by a sensor if the Euclidean distance between the point and the sensor is no more than the sensing range $r$ according to the disk/Boolean sensing model.

However, in practical scenarios, three-dimensional deployment is more feasible and realistic in ocean column monitoring, under-ground tunnels and other space applications[21]. In such cases, the area of interest needs to be studied as threedimensional space, being more representative, practical and equivalent to the real world. In three-dimensional space, the localization problem can be abstracted as a threedimensional sphere coverage issue. [22] When deploying an indoor 3D WSN, it is important to be able to determine positions of the sensor nodes that achieve the fullcoverage of the target space and the connectivity between the sensor nodes with the minimum deployment cost. The sensor node deployment problem for 3D coverage and connectivity is NP-hard even without obstacles in the target field[23]. 
Both two-dimensional and three-dimensional deployment share the probabilistic sensing model [24][25] which can be taken as an extension of the binary disc sensing model. Assume sensor $S_{i}$ is deployed at point $\left(x_{i}, y_{i}\right)$. For any point $\mathrm{P}$ at $(x, y)$, the coverage of node $S_{i}$ on point $\mathrm{P}$ can be denoted as the following formula:

$$
C_{P}\left(S_{i}\right)=\left\{\begin{array}{lr}
0 & \text { if } r+r_{i} \leq d\left(S_{i} P\right) \\
e^{-\lambda \omega^{\theta}} & \text { if } r-r_{\theta} \leq d\left(S_{i} P\right) \leq r+r_{\theta} \\
1 & \text { if } r-r_{i} \geq d\left(S_{i}+P\right)
\end{array}\right.
$$

Where $\alpha=d\left(S_{i^{*}} P\right)$ denotes the Euclidean distance between node $S_{i \mathrm{i}}$ and $\mathrm{P} ; \lambda$ and $\beta$ are the coefficients related with the sensing probability, whose values are set to be 0.5 and 1 , respectively; $r$ is the sensing range; and $r_{\varepsilon}$ is the error of sensing range. Compared with the conventional binary disk model, the probabilistic sensing model is capable of capturing the sensing uncertainties often encountered in real-world WSN applications [26].

Many of the popular placement strategies pursued for two-dimensional space become NP-Hard in three-dimensional space. With the increased interest in applications of sensor networks, such as space exploration, airborne and underwater surveillance, oceanic studies, storm tracking, deployment strategies for three-dimensional are expected in the next few years. In fact, some preliminary research results have started to emerge.

\section{Conclusion}

This paper intends to help new researchers entering the domain of WSNs by providing a comprehensive survey on deployment strategies. Sensor deployment strategies play a very important role in providing better QoS, which relates to the issue of how well each point in the sensing field is covered, each node is connected and network lifetime. However, due to severe resource constraints, hostile environmental conditions, inconvenience of positioning or high cost of nodes, it is nontrivial to design an efficient deployment strategy that would minimize cost, reduce computation, minimize node-tonode communication, and provide a high degree of area coverage, while at the same time maintaining a globally connected network is nontrivial. Challenges also arise because topological information about a sensing field is rarely available and such information may change over time in the presence of obstacles.

With a better understanding of node type and objectives of deployment, deployment strategies are discussed over their advantages and disadvantages and applicable conditions. Further work may be needed in more accurate comparison and stimulation.

\section{Acknowledgment}

This work was supported by The National Natural Science Fund, No 31670554. This work was supported by Natural Science Foundation of Jiangsu Province, Grants No BK20161527. The project sponsored by the Scientific Research Foundation for the Returned Overseas Chinese Scholars, State Education Ministry. This work was supported by TAPP: Top-notch Academic Programs Projects of Jiangsu Higher Education Institutions. The corresponding author is Fuquan Zhang. This paper is a revised and expanded version of a paper entitled, "A Survey on Deployment of Wireless Sensor Network", presented at The 5th International Conference on Information Science and Industrial Applications, Harbin, China, 2016. 


\section{References}

[1] A. Abed, A. Alkhatib, and G. S. Baicher, "Wireless Sensor Network Architecture", Int. Conf. Comput. Networks Commun. Syst., vol. 35, no. Cncs, (2012), pp. 11-15.

[2] P. Harrop and R. Das, "Wireless Sensor Networks (WSN) 2014-2024: Forecasts, Technologies, Players", IDTechEx, (2014).

[3] P. Rawat, K. D. Singh, H. Chaouchi and J. M. Bonnin, "Wireless sensor networks: A survey on recent developments and potential synergies", Journal Supercomput., vol. 68, no. 1, (2014), pp. 1-48.

[4] C. Buratti, A. Conti, D. Dardari and R. Verdone, "An overview on wireless sensor networks technology and evolution", Sensors, vol. 9, no. 9, (2009), pp. 6869-6896.

[5] K. Z. Lu, G. L. Chen, Y. H. Feng, G. Liu and R. Mao, "Approximation algorithm for minimizing relay node placement in wireless sensor networks", Sci. China Inf. Sci., vol. 53, no. 11, (2010), pp. 23322342.

[6] C. Zhu, C. Zheng, L. Shu and G. Han, "A survey on coverage and connectivity issues in wireless sensor networks", Journal of Network and Computer Applications, vol. 35, no. 2, (2012), pp. 619-632.

[7] Z. Bojkovic and B. Bakmaz, "A survey on wireless sensor networks deployment", WSEAS Trans. Commun., vol. 7, no. 12, (2008), pp. 1172-1181.

[8] R. P. S. Anju Sangwan, "Survey on Coverage in Wireless Sensor Networks Deployment", Wirel. Pers Commun, vol. 80, (2015), pp. 1475-1500.

[9] P. K. Hirani, "A Survey on Coverage Problem in Wireless Sensor Network", vol. 116, no. 2, (2015), pp. 2-4.

[10] G. Devi and R. S. Bal, "Node Deployment Coverage in Large Wireless Sensor Networks", Journal of Network Communications and Emerging Technologies, vol. 6, no. 2, (2016), pp. 19-25.

[11] H. Zhang and C. Liu, "A Review on Node Deployment of Wireless Sensor Network", vol. 9, no. 6, (2012), pp. 378-383.

[12] J. Tian, X. Liang and G. Wang, "Deployment and reallocation in mobile survivability-heterogeneous wireless sensor networks for barrier coverage", Ad Hoc Networks, vol. 000, (2015), pp. 1-11.

[13] A. Ghosh and S. Das, "Coverage and connectivity issues in wireless sensor networks: A survey", Mobile, Wireless, Sens. Networks Technol. Appl. Futur. Dir., vol. 4, no. 3, (2006), pp. 221-256.

[14] Z. Cheng, M. Perillo, W. B. Heinzelman and S. Member, "General Network Lifetime and Cost Models for Evaluating Sensor Network Deployment Strategies”, Ieee Trans. Mob. Comput., vol. 7, no. 4, (2008), pp. 484-497.

[15] J. H. Chang and L. Tassiulas, "Energy Conserving Routing in Wireless Ad-hoc Networks", Proceedings IEEE INFOCOM 2000, Conf. Comput. Commun. Ninet. Annu. Jt. Conf. IEEE Comput. Commun. Soc. Reach. Promised L. Commun., vol. 1, (2000), pp. 22-31.

[16] K. Akkaya, M. Younis and M. Bangad, "Strategies and Techniques for Node Placement in Wireless Sensor Networks: A Survey", Comput. Networks Int. Journal Comput. Telecommun. Netw., vol. 49, no. 4, (2005), pp. 512-534.

[17] S. S. Dhillon and K. Chakrabarty, "Sensor placement for effective coverage and surveillance in distributed sensor networks", IEEE Wirel. Commun. Netw. Conf. WCNC, vol. 3, no. C, (2003), pp. $1609-1614$.

[18] Z. J. Wang, X. Qian, J. F. Zhang, Y. Zhao and J. J. Guo, "Survey on node deployment in wireless sensor networks", Journal of Measurement Science and Instrumentation, vol. 03, no. 1, (2012), pp. 80-84.

[19] J. Yick, B. Mukherjee and D. Ghosal, "Wireless sensor network survey", vol. 52, (2008), pp. 2292-2330.

[20] T. Alhmiedat, "A Survey on Environmental Monitoring Systems using Wireless Sensor Networks", Journal Networks, vol. 10, no. 11, (2016), pp. 606-615.

[21] A. Somani, S. Kohli and P. P. Bhattacharya, "Comparison of Heterogeneous Leach in Two Dimensional and Three Dimensional Wireless Sensor Networks", vol. 1, no. 1, (2016), pp. 38-48.

[22] X. Hua, Z. Jinjin and B. Lei, "A NEW THREE-DIMENSION SPATIAL LOCATION", vol. 9, no. 1, (2016), pp. 233-255.

[23] M. T. Kouakou, S. Yamamoto, K. Yasumoto and M. Ito, "Deployment planning tool for indoor 3DWSNs", in Proceedings of the 12th ACM international conference adjunct papers on Ubiquitous computing, no. 1, (2010), pp. 369-370.

[24] P. Jiang, J. Liu, B. Ruan, L. Jiang and F. Wu, "A New Node Deployment and Location Dispatch Algorithm for Underwater Sensor Networks", Sensors, vol. 16, no. 1, (2016), p. 82.

[25] H. L. Wang and W. H. Chung, "The generalized k-coverage under probabilistic sensing model in sensor networks”, IEEE Wirel. Commun. Netw. Conf. WCNC, (2012), pp. 1737-1742.

[26] X. Tan, F. Zhang, K. Chen and D. Gao, "A Survey on Deployment of Wireless Sensor Network", The 5th International Conference on Information Science and Industrial Applications, (2016), Harbin, China. 


\section{Authors}

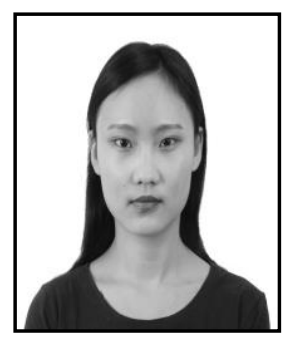

Tan Xinyi, she received an undergraduate degree in College of Information Science and Technology, Nanjing Forestry University, 2015. Her current research interests include wireless sensor network, Internet of things.

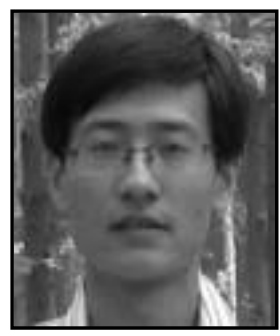

Demin Gao, he received a Ph.D. degree in 2012 from Nanjing University of Science and Technology Department of Computer Science and Engineering. In 2012, he joined in the Nanjing Forestry University as a lecturer. His research interests include routing protocols for delay tolerant, data aggregation and multiconstrained routing algorithms in wireless sensor networks and wireless sensor networks.

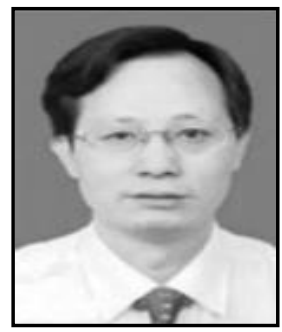

YunFei Liu, he received a Ph.D. degree from Nanjing University of Aeronautics \& Astronautic of China in 2005. He is a professor of Electronic and Communication Engineering at the College of Information Science and Technology, Nanjing Forestry University. His current research interests is signal processing technology. 
International Journal of Future Generation Communication and Networking Vol. 9, No. 11 (2016) 